\title{
Timekeeping for the Space Technology 5 (ST-5) Mission
}

\author{
Dave Raphael \\ NASA/GSFC \\ Code 561 \\ Greenbelt, MD 20771 \\ 301-286-0178 \\ David.Raphael-1@nasa.gov
}

\author{
Phil Luers \\ NASA/GSFC \\ Code 561 \\ Greenbelt, MD 20771 \\ 301-286-5777 \\ Philip.J.Luers@nasa.gov
}

\author{
Victor Sank \\ GSFC/QSS Group, Inc. \\ Code 567 \\ Greenbelt, MD 20771 \\ 301-286-2645 \\ Victor.J.Sank@gsfc.nasa.gov
}

\author{
George Jackson \\ NASA/GSFC \\ Code 561 \\ Greenbelt, MD 20771 \\ 301-286-3255 \\ George.L.Jackson@nasa.gov
}

Abstract - Space Technology 5, or better known as ST-5, is a space technology development mission in the New Millennium Program (NMP) and NASA's first experiment in the design of miniaturized satellite constellations. The mission will design, integrate and launch multiple spacecraft into an orbit high above the Earth's protective magnetic field known as the magnetosphere. Each spacecraft incorporates innovative technology and constellation concepts which will be instrumental in future space science missions. A total of three ST-5 spacecraft will be launched as "secondary" payloads into a highly elliptical geosynchronous transfer orbit, and will operate as a 3-element constellation for a minimum duration of 90 days.

In order to correlate the time of science measurements with orbit position relative to the Earth, orbit position in space (with respect to other objects in space) and/or with events measured on Earth or other spacecraft, accurate knowledge of spacecraft and ground time is needed. Ground time as used in the USA (known as Universal Time Coordinated or UTC) is maintained by the U.S. Naval Observatory. Spacecraft time is maintained onboard within the Command and Data Handling (C\&DH) system. The science requirements for ST-5 are that spacecraft time and ground time be correlatable to each other, with some degree of accuracy.

Accurate knowledge of UTC time on a spacecraft is required so that science measurements can be correlated with orbit position relative to the Earth, orbit position in space and with events measured on Earth or other spacecraft. The most crucial parameter is not the clock oscillator frequency, but more importantly, how the clock oscillator frequency varies with time or temperature (clock oscillator drift). Even with an incorrect clock oscillator frequency, if there were no drift, the frequency could be assessed by comparing the spacecraft clock to a ground clock during a few correlation events. Once the frequency is accurately known, it is easy enough to make a regular adjustment to the spacecraft clock or to calculate the correct ground time for a given spacecraft clock time. The oscillator frequency, however, is temperature dependent, drifts with age and is affected by radiation; hence, repeated correlation measurements are required.

\section{TABLE OF CONTENTS}

1 INTRODUCTION

2 ST-5 MISSION OVERVIEW

3 NeW MillenNiUm Program TeChNOlogies

4 TIMING REQUIREMENTS

4.1 Accuracy Requirement

4.2 Knowledge Requirement

4.3 Origin of Timing Requirement

4.3.1 Science Rationale

4.3.2 Incremental Time with Respect to

Science Data

4.3.3 Navigation and Tracking Time

Requirement

4.3.4 Data Time Tagging Requirements

5 RETURN DATA DELAY (RDD) TIME CORRELATION METHOD

6. SPACECRAFT IMPLEMENTATION

6.1 C\&DH Hardware

6.2 Flight Software

6.2.1 Spacecraft Universal Time (S/C UT)

6.2.2 UT Correction Factor (UTCF)

6.2.3 Leap Seconds

6.2.4 $1 \mathrm{~Hz}$ Adjust Mechanism

6.2.5 Onboard Time Management

7. ST-5 CORRELATION METHOD

7.1 One Way Method: Return Data Delay (RDD)

7.1.1 Basic Concept, Return Data Delay

Method

8. GRound Station TimeKEEPING

9. CONCLUSION

10. ACRONYM LIST

11. BIOGRAPHIES

1 0-7803-7651-X/03/\$17.00 02003 IEEE

${ }^{2}$ IEEEAC paper \#1418, Updated December 3, 2002 


\section{INTRODUCTION}

The C\&DH hardware will meet the following requirements for ST-5:

- Time correlation knowledge between two spacecraft shall be less than 10 milliseconds ( $\mathrm{ms}$ ).

- Time correlation knowledge between spacecraft Universal Time and UTC shall be less than $5 \mathrm{~ms}$.

- Time correlation data frames from the spacecraft shall be time tagged to an accuracy of at least $0.5 \mathrm{~ms}$ of onboard spacecraft time.

- Spacecraft Universal Time vs. UTC shall be less than or equal to 1 second over one complete orbital period over normal operational temperature ranges.

- Temperature measurements of the onboard clock shall be telemetered to the ground to facilitate clock stability calculations.

- Time tagging of science validation data shall have a resolution of at least $0.1 \mathrm{~ms}$.

- The spacecraft shall provide the ability to update and adjust the onboard clock.

This paper provides an overview of the method that will be used to determine the difference or error between the spacecraft and ground, and also maintenance methods for keeping the spacecraft within specifications.

The C\&DH hardware uses a $24 \mathrm{MegaHertz}(\mathrm{MHz})$ oscillator as baseline and the design for the spacecraft timer is incorporated into an Actel 54SX32 Field Programmable Gate Array (FPGA).

\section{ST-5 MISSION OVERVIEW}

The Space Technology 5 mission is a part of NASA's New Millennium Program (NMP). The ST-5 project is an inhouse development managed at the Goddard Space Flight Center in Greenbelt, Maryland. A primary goal of ST-5 is to provide a proof-of-concept demonstration for future nano-satellite constellation missions. The project will accomplish this by developing and flying a satellite constellation comprised of three small, low-power, fullservice spacecraft, each with a total mass of about 25 kilograms $(\mathrm{kg})$. During the three-month mission, each of the spacecraft will demonstrate several NMP nano-satellite and constellation-enabling technologies. Major goals include showing that multiple spacecraft can operate together as a single constellation (and not just a group of individual units) and showing that a very small spacecraft is capable of research-quality science measurements.

Each 25-kg spin-stabilized spacecraft has a total power budget of approximately 22 watts, which is provided by high-efficiency, triple-junction solar cell arrays and a lithium-ion battery. The three ST-5 spacecraft will be launched as "secondary" payloads into a highly elliptical geo-synchronous transfer orbit, with a period of about 10.5 hours, a perigee distance of about 220 kilometers $(\mathrm{km})$ and an apogee distance of about $38,000 \mathrm{~km}$. All three will share the same orbit plane, and the separation distance between spacecraft near apogee is expected to be between 100 and $1,000 \mathrm{~km}$. The spacecraft spin rate will be about 20 revolutions per minute (RPM).

\section{New MillenNiUm Program TeChNologies}

The purpose of NASA's New Millennium Program is to develop and flight validate the critical "breakthrough" technologies that are needed to enable the space science and Earth science missions of the future. For ST-5, the focus is on the small, low-mass and low-power theme. These technologies include a miniature and low-power X-band transponder for uplink and downlink, a cold gas microthruster (CGMT) propulsion system, two variable emittance controllers (VEC) for the thermal subsystem, "flex" harness interconnects for the solar panels, and a new type of $1 / 2$ volt CMOS ultra low-power, radiation-tolerant (CULPRiT) logic. The $1 / 2$ volt CULPRiT technology, which is latchup immune and hardened for up to 100 kilorads (krads) of total ionization dose, will be verified as an integral part of the C\&DH subsystem.

\section{TIMING REQUIREMENTS}

\subsection{Accuracy Requirement}

Spacecraft (S/C) Universal Time vs. UTC shall be less than or equal to 1 second over one complete orbital period over normal operational temperature ranges.

The ST-5 S/C time shall never differ from UTC by more than 1 second. Since the ST-5 S/C is without ground contact for the majority of the time, the S/C clock drift must be sufficiently small that this requirement is met at all times during the ST-5 orbit. Measuring, correlating and correcting the spacecraft time may be performed during every ground pass.

\subsection{Knowledge Requirement}

Time correlation knowledge between two spacecraft shall be less than 10 milliseconds (ms). Time correlation knowledge between spacecraft Universal Time and UTC shall be less than $5 \mathrm{~ms}$.

The ST-5 individual spacecraft times shall be correlated to one another with an accuracy of $10 \mathrm{~ms}$. In lieu of interspacecraft communication, the derived requirement shall be: The ST-5 S/C time shall be correlatable to UTC with an accuracy of $5 \mathrm{~ms}$.

\subsection{Origin of Timing Requirement}




\subsubsection{Science Rationale}

Science Requirement

1. "Science events" (MHD waves, magnetic field variations, energetic particle injection fronts, etc.) all propagate at the local Alfven speed $\sim 1,000$ to 5,000 kilometers per second $(\mathrm{km} / \mathrm{s})$ at ST-5 altitudes.

2. ST-5 inter-S/C spacing will be $\sim 100 \mathrm{~km}$.

3. Time difference between the arrival of science events amongst the 3 spacecraft will be $\sim 20$ to $100 \mathrm{~ms}$.

4. To determine propagation characteristics (k-vectors polarization, phase speed, etc.) for these science events, the accuracy of the magnetic field time tagging at each spacecraft (relative to each other) should be $1 / 10$ th of 20-100 ms (i.e., 2-10 ms).

Impact of reduced accuracy requirement:

1. With better than $10 \mathrm{~ms}$ timing accuracy, ST-5 may make some important new "discoveries" relating to the dynamics of the inner magnetosphere.

2. Between $10-100 \mathrm{~ms}$ accuracy, there is hope that the data will still be meaningful.

3. After $100 \mathrm{~ms}$, it may not be possible to really intercompare the measurements for many scientific purposes in a meaningful manner.

\subsubsection{Incremental Time with Respect to Science Data}

Assumptions will be made based on some of the characteristics of the Wind electron and ion detector. Clock correlation with UTC will be discussed in a later section.

There is an assumption that as the spacecraft spins, particle collection data will be binned based in units of $30^{\circ}$, i.e. each rotation is broken into 12 regions of space. Since the clock (counter) will drift, pointing direction cannot depend on it alone, however, since data from a given section of space is likely to be average over only a few minutes, the angular error will be of the order of $(120 \mathrm{~s}) \times\left(1 \times 10^{-6}\right) \times\left(120^{\circ} / \mathrm{s}\right)=$ 14.4 millidegrees in 2 minutes, which is insignificant. If the sector size were much less than $30^{\circ}$, this accuracy would need to be re-evaluated and the number of clock pulses per rotation (based on Sun pulse) may need to be updated more often.

Over the long term, the direction in space that is allocated to each bin will drift if all that is used is the clock to control the data binning. Based on the 1 part per million (ppm), clock drift rate and $120^{\circ} / \mathrm{s}$ spin rate, a $30^{\circ}$ error will occur after $\left(30^{\circ}\right) /\left(120 \mu^{\circ} / \mathrm{s}\right)=250,000 \mathrm{~s}=2.9$ days. If the clock is not updated or compared to the attitude sensor (Sun pulse), this precession of bin positions relative to an inertial (nonrotating) coordinate system will occur. Updating the number on clock counts per rotation will solve the problem. A clock correlation table will need to be maintained on the ground for each spacecraft. A separate table containing attitude sensor data as a function of clock count will also be required. As an alternative, the rotation rate and phase can be maintained as a function of UTC.
The assumption of the 2-minute data collection cycle relates to another parameter, orbit position accuracy. The perigee velocity for a GTO orbit is $10.25 \mathrm{~km} / \mathrm{s}$ and the apogee velocity is $1.60 \mathrm{~km} / \mathrm{s}$ relative to the earth. At apogee, the spacecraft will travel $(1.6 \mathrm{~km} / \mathrm{s}) \times(20 \mathrm{~s})=32 \mathrm{~km}$ during one data collection cycle and at every other point in the orbit, it will travel further, up to $(10.25 \mathrm{~km} / \mathrm{s}) \times(120 \mathrm{~s})=1230 \mathrm{~km}$ at perigee. The scientist will have to decide if this meets the science requirement, the purpose here is to realize that a 50 $\mathrm{km}$ or greater orbit position, error may be well within the science requirement especially for missions that map large regions of space.

Another parameter to consider is earth's velocity around the sun, which is about $30 \mathrm{~km} / \mathrm{s}$. For mapping the earth's magnetosphere which is attached to the earth, this velocity may not be a factor but it is large compared to the satellite velocity relative to the earth.

\subsubsection{Navigation and Tracking Time Requirement}

Orbit determination is done on the ground and does not make use of the onboard clock. Although attitude determination is also done on the ground, it makes use of observations made on board that make measurements of the Sun and the Earth's magnetic field. A spacecraft time tag is attached to these measurements and the information is telemetered to the ground, to be used for this processing.

\subsubsection{Data Time Tagging Requirements}

Time tagging will be provided for the following:

1. Sun Crossing

2. Magnetometer Vector

3. Downlink

4. Every $1 \mathrm{sec}$ interval

5. $\mathrm{S} / \mathrm{W}$ Trigger

6. External Trigger

\section{Return Data Delay (RDD) Time CORRELATION METHOD}

The one way method of return data delay is reasonably simple and most common because it has the minimum equipment requirement, however its accuracy is limited and it is prone to mistakes when an attempt is made to use it for accuracy better than about $1 \mathrm{~ms}$. In this method, a specific event in the downlink stream is time tagged on the spacecraft, and its ground receipt time is recorded by the ground station. Comparing these two times, taking into account delays through hardware and the actual transmission time, will indicate how far ahead or behind UTC the ST-5 spacecraft is.

\section{SPACECRAFT IMPLEMENTATION}




\subsection{C\&DH Hardware}

There are $31,557,600$ seconds in an average year $(24 \mathrm{x}$ $3,600 \times 365.25=31,557,600$ ) requiring a minimum of 25 bits $\left(2^{25}=33,554,432\right)$ to represent seconds. For a granulation of 1 microsecond $(\mu \mathrm{s})$ in the sub-seconds portion of the time, a minimum of 20 bits are required, hence a minimum of 45 bits are required.

The C\&DH Mission Elapsed Timer (MET), as its name suggests, provides an ongoing count of the actual time elapsed on the spacecraft. This counter is combined with the software-loaded offset timing number in a processor memory location to represent the actual UTC time.

The MET consists of a 32-bit up-counter clocked by a $1 \mathrm{~Hz}$ clock input. This provides sufficient capability to count up to $2^{32}$ seconds $(4,294,967,296$ seconds $=49,710$ days or approximately 136 years) before the MET counter rolls over. The MET seconds is cleared only during a board power-up reset. In addition, the MET can be "jammed" with a 32-bit value by the flight software via ground command to set the mission elapsed timer to the desired initialization value. Jamming the MET register is useful for setting the MET to a specified value for troubleshooting and test purposes, as well as for reinstating the previous MET in the event that the MET register is cleared due to a power-on reset while in flight.

The Sub-Seconds Timer (SST), like the MET, keeps track of elapsed time by keeping a sub-seconds time count. The subseconds count can be latched and stored at certain events, the value being used by the spacecraft to time tag specific data and events. The SST consists of a 22-bit up-counter clocked by a $1 \mathrm{MHz}$ clock input. This provides the $\mathrm{C} \& \mathrm{DH}$ board with the ability to keep track of sub-seconds time at a resolution of $1 \mu \mathrm{sec}$. The SST counts up to its maximum value of approximately 4.194 seconds before rolling over to zero and continuing its count.

The spacecraft hardware time is composed of the sum of the seconds counter and the sub-seconds counter.

In order to perform the RDD correlation described above, the C\&DH will latch the seconds and sub-seconds time of the transmission of every 16th Virtual Channel 0 (VCO mod16) telemetry frame. The VC0 mod 16 frame will occur approximately every 162 seconds $(252 \times 8 \times 5+32$ bits/frame $\times 16$ frames $\times 1 \mathrm{~ms} / \mathrm{bit}=161.792 \mathrm{~s}$ ). The flight software will gather the latched hardware time of the transmission of the frame, and calculate the S/C Universal Time of the transmission of the VC0 modl 6 frame.

A block diagram of the Time Circuit is shown in Figure 1 and Figure 2. Figure 1 shows how the value of the MET is sampled and stored in one of two distinct I/O-mapped 16-bit registers (bits 15:0) under the following conditions:

1. The MET value is latched and stored in the "Sun Crossing Time Latch" in response to a Sun Crossing pulse.

2. The MET value is latched and stored in the "Downlink Time Correlation Trigger Latch" in response to a Downlink Time Correlation pulse.

It also shows how the value of the SST is sampled and stored in one of five distinct I/O-mapped 22-bit registers (bits 21:0) under the following event conditions:

1. The SST value is latched by the $1 \mathrm{~Hz}$ MET clock input and stored in the "1 Second Latch" register every second.

2. The SST value is latched and stored in the "Software-Triggered Latch" register when there is a software memory write.

3. The SST value is latched and stored in the "External Trigger Latch" upon receipt of an active low pulsed signal from the C\&DH test connector.

4. The SST value is latched and stored in the "Sun Crossing Time Latch" in response to a Sun Crossing pulse.

5. The SST value is latched and stored in the "Downlink Time Correlation Trigger Latch" in response to a Downlink Time Correlation pulse.

Figure 2 illustrates how the Magnetometer (MAG) time tagging is transferred from one FPGA to the next. The MET and SST values are stored in the "MAG Vector Trigger Latch" in response to a MAG Time Tag Latch. The 48 bits of time tag are transferred a byte at a time.

\subsection{Flight Software \\ 6.2.1 Spacecraft Universal Time (S/C UT)}

The S/C UT is the sum of three values: the Mission Elapsed Time (MET), the UT Correction Factor (UTCF), and the leap seconds. S/C UT has a resolution of 1 microsecond and is used for stored command processing.

Because of the complexity of storing numbers like 12:00:00.000 Noon 13 February, 2001 or 00-04417:00:00.000 GMT, times on the spacecraft are stored as a number of seconds since an arbitrarily chosen epoch. For ST-5, the epoch has been chosen as 1 January 2000 12:00 GMT. The S/C UT will be stored as a number of seconds and sub-seconds since this mission epoch.

The first part of the S/C UT is the Mission Elapsed Time (MET) counter. This telemetry point is incremented once per second by a $1 \mathrm{~Hz}$ clock input. The MET may be jammed via ground command at any time, most notably it may be jammed with a value upon spacecraft power-up. 


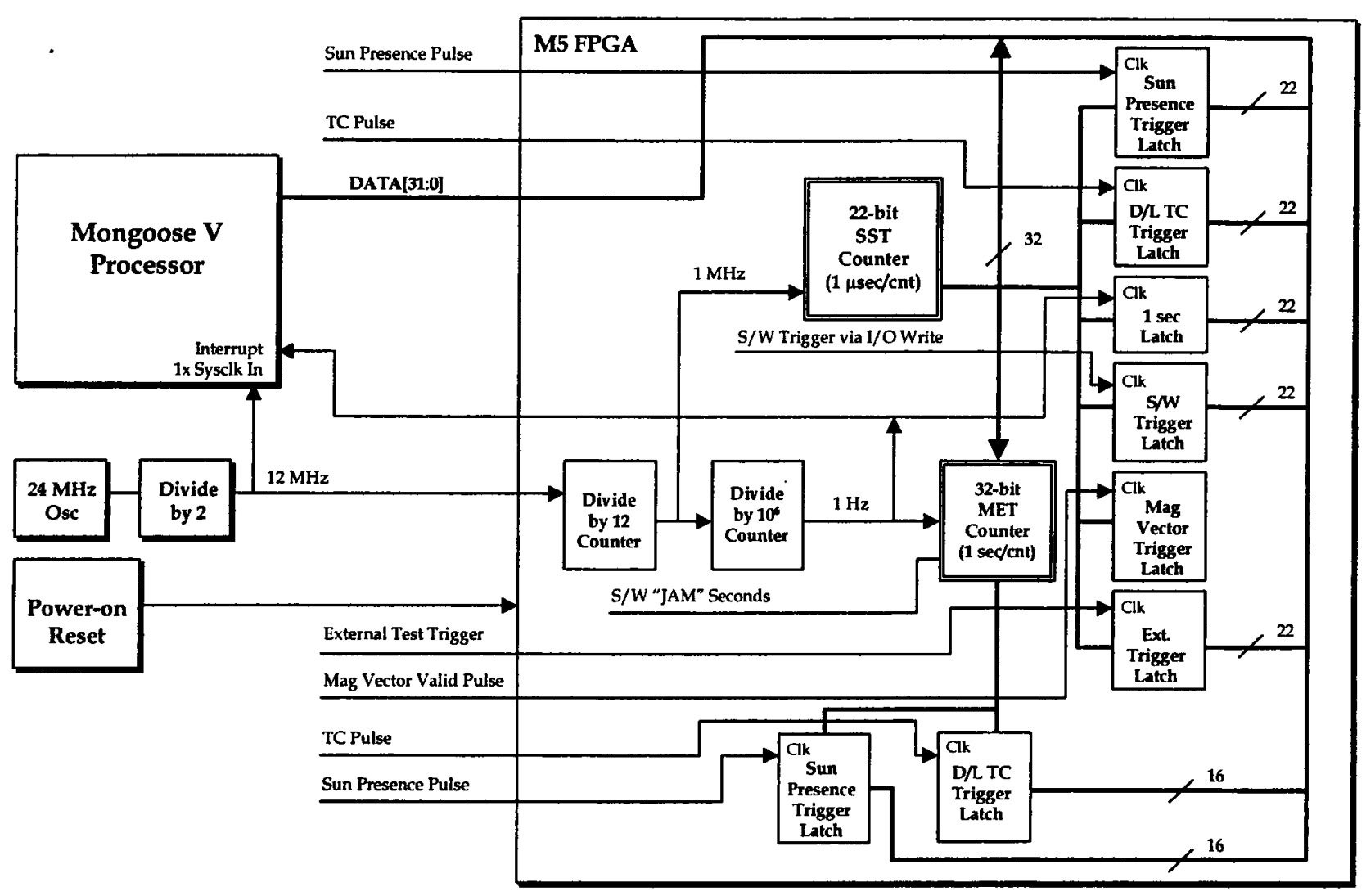

Figure 1 - Time Circuit Block Diagram (1 of 2)

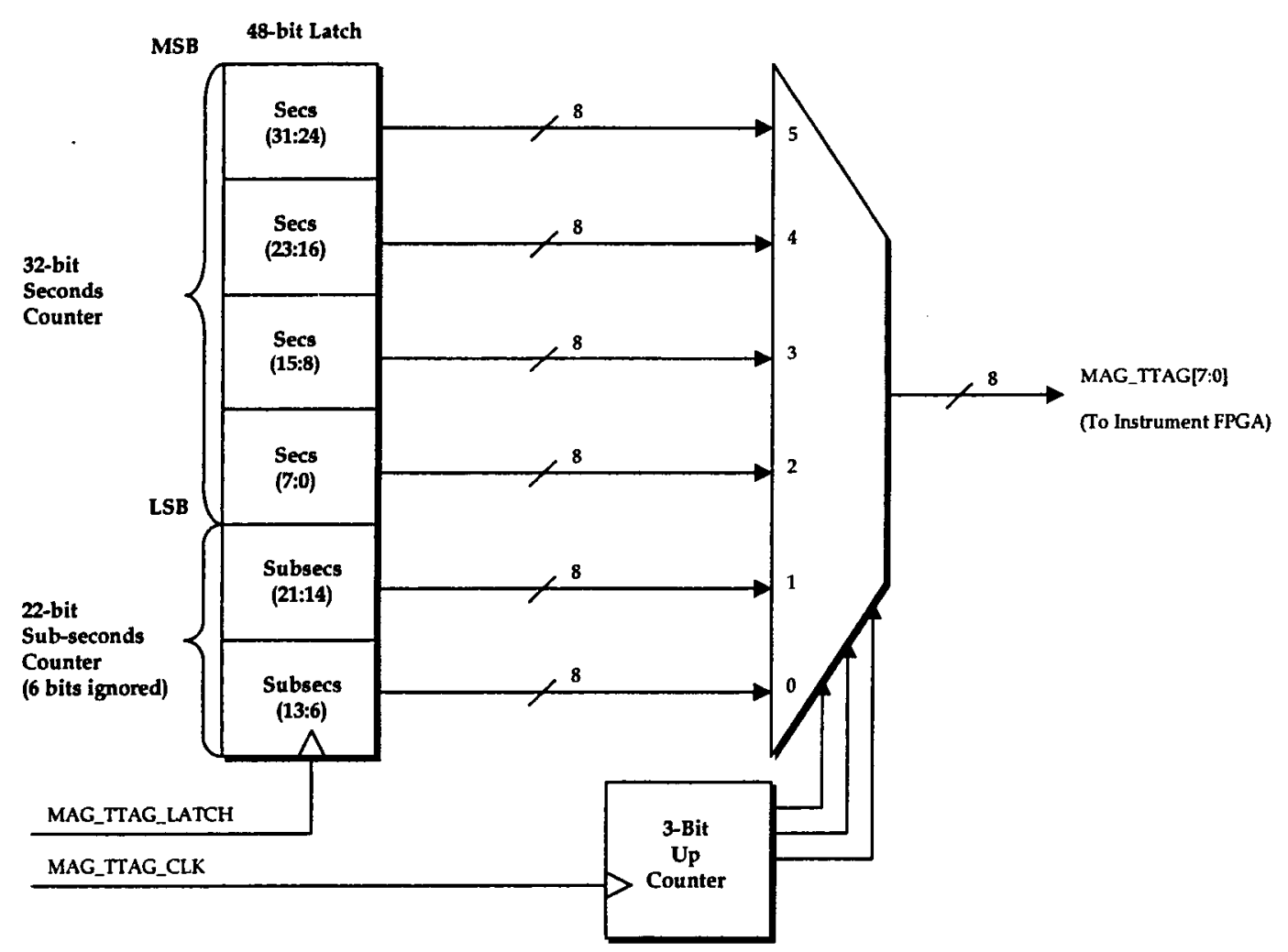

Figure 2 - Time Circuit Block Diagram (2 of 2) 


\subsubsection{UT Correction Factor (UTCF)}

In order that the spacecraft time be adjustable, and that the MET not have discontinuities, the spacecraft maintains a ground commandable correction factor, the UTCF. The UTCF is a static value, currently an unsigned integer only adjusted by ground command. Because of this, it will be initialized to a large positive value, most likely 4 years if the launch is in late 2003. If/when the spacecraft drifts ahead of ground time, the UTCF will be decreased via ground command. If/when the spacecraft drifts behind ground time, the UTCF will be increased. The UTCF may be adjusted, via ground command, at any time during the mission to keep the S/C UT within 1 second of UTC.

\subsubsection{Leap Seconds}

In order to keep the cumulative difference in UT1 (astronomical time) and UTC less than 0.9 seconds, a leap second is added to the atomic time to decrease the difference between the two. This leap second can be either positive or negative depending on the Earth's rotation. Since the first leap second in 1972, all leap seconds have been positive and there were 22 leap seconds in the 27 years to January, 1999. This pattern reflects the general slowing trend of the Earth due to tidal braking. (http://tycho.usno.navy.mil/leapsec.html)

Since 1972, there has been 22 leap seconds, happening at irregular intervals (as little as 6 months in 1972 and as long as 2.5 years from July 1985 to January 1988). The USNO has posted months in advance a notice when leap seconds will occur, and also when they have determined that they won't occur.

Since the ST-5 mission is using UTC as its time reference, the spacecraft must keep up with the leap seconds adjustments made by the USNO. The Flight Software (FSW) maintains a telemetry point that reflects this. The spacecraft leap seconds may be commanded from the ground at any time during the mission, and most likely this will happen whenever the USNO decides that a leap second is necessary.

\subsubsection{Hz Adjust Mechanism}

The C\&DH Flight Software includes a mechanism for compensating for a $\mathrm{C} \& D H$ clock oscillator that is slightly fast or slightly slow, resulting in an overall clock drift. Clock drift with respect to UTC basically means that every time that the C\&DH hardware indicates a second has elapsed, actually a time slightly smaller or slightly larger than a second has passed. A ground commandable parameter, the $1 \mathrm{~Hz}$ adjustment factor can be applied to the MET to correct for clock drift. The Flight Software, when reading the MET, multiplies the difference between the last value and the new value by the $1 \mathrm{~Hz}$ adjustment factor every second to reduce clock drift.

\subsubsection{Onboard Time Management}

Time is maintained in the FSW in Mission Elapsed Time (MET) and Universal Time (UT) formats. The UT format is needed for stored command management and is calculated on board by adjusting the MET, adding the Universal Time Correction Factor (UTCF) and the Leap Seconds once per second.

S/C UT $=1 \mathrm{~Hz}$ Adjustment Factor x MET + UTCF + Leap Seconds

A startup sequence that results in a correctly configured S/C clock may be something like:

1. Set the seconds portion of the MET (jam occurs on 1 sec mark)

2. Set UTCF (jam UTCF)

3. Adjust UTCF by adding or subtracting a specified value $<2 \mathrm{~ms}$ (adjust UTCF)

4. Set a $1 \mathrm{~Hz}$ adjustment factor to a specified positive or negative value

The ST-5 spacecraft maintains time in a combination of both hardware and software. The Flight Software uses the hardware counters, the MET, the UTCF and the leap seconds to represent one single value, the S/C UT. Mechanisms exist for coarse adjustment of S/C UT, fine adjustment of S/C UT and constant adjustment to reduce S/C UT drift with respect to UTC. The hardware supports the S/C UT to UTC correlation method that will meet the overall mission time accuracy requirements.

\section{ST-5 CORRELATION METHOD}

\subsection{One Way Method: Return Data Delay (RDD)}

RDD is an open loop method and is also called the Return Channel Time Delay (RCTD) method of clock correlation. The spacecraft records S/C UT of the VC0 mod 16 telemetry frames. The ground system places UTC time tags on the frames as they arrive at the ground (ground receipt time, GRT). It is then a simple matter to subtract the various delays to determine the difference between S/C UT and UTC.

\subsubsection{Basic Concept, Return Data Delay Method}

The basic concept of RDD is as follows:

1. Time tag data as it leaves the spacecraft with a spacecraft clock reading.

2. Time tag the data with a ground UTC value when the data is received on the ground.

3. Using orbit knowledge, calculate the transit time from spacecraft to ground including equipment delays and subtract this value from the ground receipt time. The result is the time that the data left the spacecraft, based on UTC. 
4. Compare the result with the spacecraft clock reading in the data.

Spacecraft range, or range propagation delay, must be known for the specific time of the transit of the telemetry carrying the clock reading. This delay is reported by the Flight Dynamics Facility (FDF) in a product called a "range file." The range to the spacecraft at the time that the beginning of the frame marker left the spacecraft antenna must be used.

Satellites using the NASA ground network use the one way RDD method for their clock correlation. The GN, DSN, SN (Space Network, not used by ST-5) and commercial networks place a time tag on spacecraft telemetry data as it is received on the ground (GRT) to an accurate to $1 \mathrm{~ms}$ or better.

In order to communicate with a satellite, we need to know where it is at a certain time so that a ground antenna can be pointed. In addition, orbit knowledge allows the ground receive system to anticipate the doppler offset of the satellites carrier frequency so the ground receivers local oscillators (LOs) can be set to compensate for the offset which then allows the receiver to find and lock to the signal in a reasonable amount of time. When the signal from the satellite is weak, a large high gain ground antenna with a narrow beamwidth is required. This implies that accurate orbit knowledge is required on the ground and the antenna pointing requirement may be the limiting factor that sets the orbit accuracy requirement. This is not relieved or aided by GPS on the satellite or by satellite autonomy. In order to properly point an 11-meter antenna to a satellite at $5 \mathrm{Re}$ using an X-Band communication signal $(\sim 8 \mathrm{GHz}$, beamwidth of $\pm 0.1^{\circ}$ ), it is required that we know the orbit position to about $60 \mathrm{~km}$. This orbit accuracy parameter is a major contributor to the absolute time correlation accuracy allowing us to now discuss mission time correlation.

As a frame of data leaves the C\&DH or data recorder on its way to the transmitter, the beginning of the first bit of the frame synchronization marker is used to trigger the latching of the MET. The S/C UT of the VC0 mod16 frame is later sent to the ground (could be 1 second or 1 day later). On the ground, a clock that is tied to UTC records the arrival time of the data, allowing us to determine the arrival time of the first bit of the Consultative Committee on Space Data Systems (CCSDS) frame, the bit that triggered the onboard MET reading.

The ground time tagging is usually to an arbitrary data bit at a regular but not necessarily periodic rate. This is called a ground receipt time (GRT). On the ground, NASCOM blocks or internet protocol (IP) packets may be used to transport the data to the science center. The first bit of every $1 / 100$ th ground packet may receive a ground time tag in a special field that is part of the packet. Since the ground packet length will not in general be the same as the spacecraft telemetry frame length, the bit that receives the GRT is arbitrary relative to the beginning of frame.

At the spacecraft operations center, knowledge of the occasional GRT allows interpolation so that the GRT of the first bit of each CCSDS frame marker (FM) can be calculated. Knowledge of the orbit allows the calculation of the position of the spacecraft when the signal was emitted and thus allows the radio frequency (RF) propagation time to be calculated. By subtracting the RF propagation time and equipment delays from the GRT for a particular (CCSDS) frame, we have the time, based on ground UTC, that this frame left the spacecraft. After subtracting the spacecraft transmitter and associated delays (convolutional coding, Reed-Solomon coding, etc.), we have the time, based on UTC, that the spacecraft clock was read.

The first delay that the VCO mod 16 encounters in getting to the ground is the delay through the remainder of the $C \& D H$ downlink circuitry, including the Reed-Solomon encoder, the Cyclic Redundancy Check (CRC), and the CCSDS randomizer which exclusive ORs the data with a $\mathrm{PN}$ pattern (if in use). In the diagram below, this time is collectively called $t_{C \& D H}$. Note that $t_{C \& D H}$ will definitely vary with telemetry rate, and will have to be determined through analysis and testing prior to launch.

The second delay that the frame encounters is the delay through the RF system. The RF cables and antenna themselves are negligible, however the modulation circuits within the X-Band transponder will have a measurable effect on the telemetry. In the diagram below, this is called $t_{X P N D R}$. Note that $t_{X P N D R}$ will vary with telemetry rate, and will have to be determined through analysis and testing prior to launch.

The next delay that the frame encounters is that of the actual transmission between the spacecraft and the Earth. In the diagram below, this is called $t_{\text {TRANSMIT. }}$. This delay must be determined during the ground contact as it will vary from orbit to orbit. However, given the ST-5 timing requirement of $5 \mathrm{~ms}$, an error of $\mathrm{d}=\mathrm{c} \times \mathrm{t}=3 \times 10^{\mathrm{s}} \mathrm{km} / \mathrm{s} \times 0.005 \mathrm{sec}=$ $1500 \mathrm{~km}$ can be tolerated.

Finally the frame arrives at the ground station and is formed into a larger ground transfer block. The ground station records the receipt time of the first bit of the data portion of the block and inserts this into the block header. In the diagram below, this is called $t_{U T C}$ and it is generated for every ground transfer block.

The VCO mod16 frame is located somewhere within the ground transfer block; this will vary from block to block. The ground station software must locate the VC0 mod16 frame within the ground transfer block and determine how many bits are between the VCO mod 16 frame and the first bit of the frame (the bit that is time-tagged as $t_{U T C}$ ). The number of bits divided by the bit rate will give you the offset in time to the VCO modl 6 frame. In the above 
VCO frame with 4 LSBs of sequence count $=0000$

(VCO mod 16 frame)

every 161 seconds (for $1 \mathrm{kbit} / \mathrm{s} \mathrm{H} / \mathrm{K}$ )

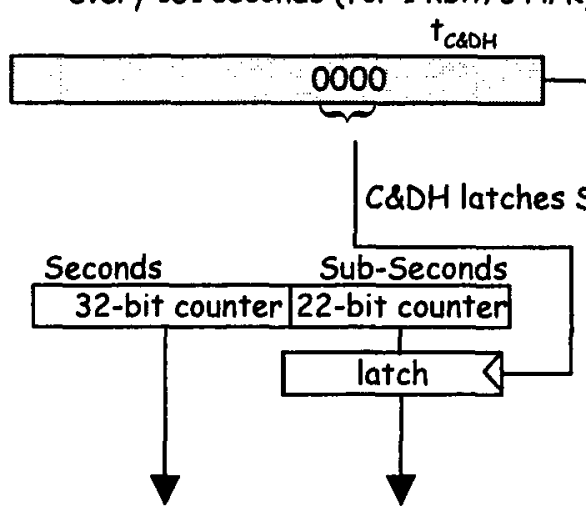

FSW reads $V C O$ mod16 frame time and forms telemetry $t_{v c o}$ reported to ground in time packet

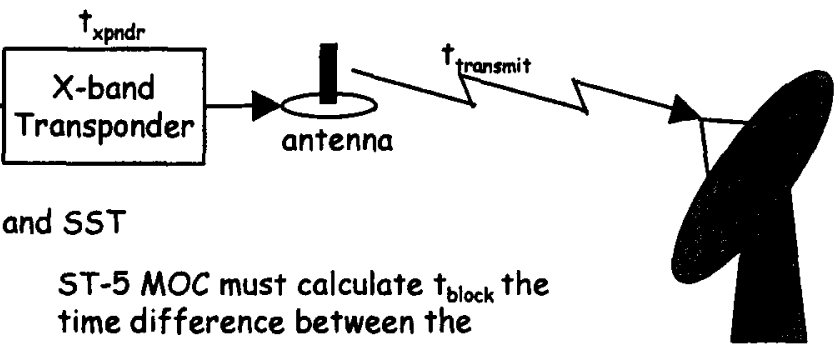
time difference between the beginning of the 4800-bit block and the VCO mod 16 frame that was time-tagged by the spacecraft.

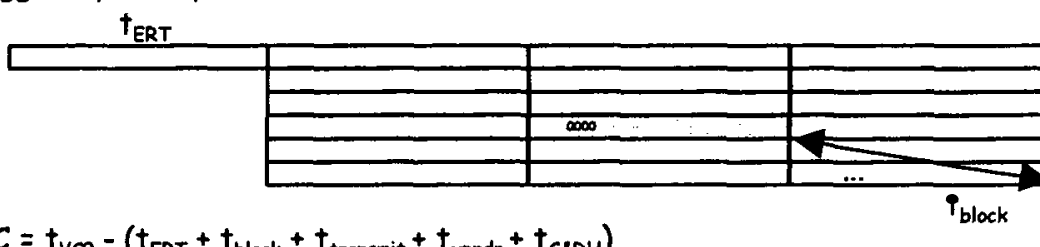

Ground Station forms 4800-bit block (SFDU) and generates time $t_{\text {ERT. }}$ the time that the trailing edge of the last bit of the 4800bit block was received.

Error $=S / C U T-U T C=t_{V C O}-\left(t_{\text {ERT }}+t_{\text {block }}+t_{\text {transmit }}+t_{\text {xpondr }}+t_{C \text { CSOH }}\right)$

diagram, this is referred to as $t_{\text {BLOCK, }}$ and will vary with telemetry rate.

Finally, the ground station must perform the math to correlate the block time tag, tUTC with the S/C UT of transmission for the frame, $t_{\mathrm{vc} 0}$.

Error $=\mathrm{S} / \mathrm{C}$ UT $-\mathrm{UTC}=\mathrm{t}_{\mathrm{VCO}}-\left(\mathrm{t}_{\mathrm{UTC}}+\mathrm{t}_{\mathrm{BLOCK}}+\mathrm{t}_{\text {TRANSMTT }}+\right.$ $\left.t_{X P N D R}+t_{C \& D H}\right)$

Where:

$t_{\mathrm{VC}}$ is reported by flight software for every VC0 $\bmod 16$ frame, varies from frame to frame.

$t_{\text {UTC }}$ is generated by the ground station for every ground transfer block, varies for every block.

$t_{B L O C K}$ is calculated by locating the VC0 mod16 frame within the ground transfer block, varies from frame to frame.

$t_{\text {TRANSMIT }}$ is reported by the ground station in the form of ranging information, varies for each ground station contact, or perhaps during the contact.

$t_{X P N D R}$ is determined by the transponder manufacturer prior to delivery, varies with telemetry bit-rate, but fixed otherwise (2 fixed values, one for 1 kilobit per second (kbps) and another for $100 \mathrm{kbps}$ )

$t_{C \& D H}$ is determined by the C\&DH design team prior to delivery, varies with telemetry bit rate, but fixed otherwise ( 2 fixed values, one for $1 \mathrm{kbps}$ and another for $100 \mathrm{kbps}$ )

\section{Ground Station TimekeEPING}

The ST-5 ground station will generate time tags for all of the telemetry received from the ST-5 spacecraft. This time tag will be forwarded in the block headers from the ground station to the Mission Operations Center (MOC). Generally, the ground station will assemble a block of data, and then record in the block header the UTC for the receipt of the first bit of data. The time in the block header combined with the telemetry bit-rate allows us to calculate the time of receipt of every bit in the telemetry stream.

\section{CONCLUSION}

The ST-5 timekeeping requirements are all very realistic with the large amount of reuse of ground hardware and flight software. However, the C\&DH, the Flight Software and the Ground Systems must all be very well coordinated in order to meet all the requirements. 


\section{ACRONYM LIST}

\begin{tabular}{|c|c|c|}
\hline CCsDS & $=$ & $\begin{array}{l}\text { Consultative Committee on Space Data } \\
\text { Systems }\end{array}$ \\
\hline C\&DH & $=$ & Command \& Data Handling \\
\hline CGMT & $=$ & Cold Gas Micro-Thruster \\
\hline CMOS & $=$ & $\begin{array}{l}\text { Complimentary Metal Oxide } \\
\text { Semiconductor }\end{array}$ \\
\hline CRC & $=$ & Cyclic Redundancy Check \\
\hline CULPRiT & $=$ & $\begin{array}{l}\text { CMOS Ultra Low-Power Radiation- } \\
\text { Tolerant }\end{array}$ \\
\hline DSN & $=$ & Deep Space Network \\
\hline FDF & $=$ & Flight Dynamics Facility \\
\hline FPGA & $=$ & Field Programmable Gate Array \\
\hline FSW & $=$ & Flight Software \\
\hline $\mathrm{GHz}$ & $=$ & GigaHertz \\
\hline GMT & $=$ & Greenwich Mean Time \\
\hline GN & $=$ & Ground Network \\
\hline GPS & $=$ & Global Positioning System \\
\hline GRT & $=$ & Ground Receipt Time \\
\hline GSFC & $=$ & Goddard Space Flight Center \\
\hline GTO & $=$ & Geosynchronous Transfer Orbit \\
\hline $\mathrm{Hz}$ & $=$ & Hertz \\
\hline kbps & $=$ & kilobits per second \\
\hline $\mathrm{kg}$ & $=$ & kilogram \\
\hline km & $=$ & kilometer \\
\hline $\mathrm{km} / \mathrm{s}$ & $=$ & kilometer per second \\
\hline MAG & $=$ & Magnetometer \\
\hline MET & $=$ & Mission Elapsed Time (or Timer) \\
\hline $\mathrm{MHz}$ & $=$ & MegaHertz \\
\hline MOC & $=$ & Mission Operations Center \\
\hline $\mathrm{ms}$ & $=$ & millisecond \\
\hline NASA & $=$ & $\begin{array}{l}\text { National Aeronautics and Space } \\
\text { Administration }\end{array}$ \\
\hline NASCOM & $=$ & NASA Communications \\
\hline NMP & $=$ & New Millennium Program \\
\hline NRZ-L & $=$ & Non-Return to Zero Level \\
\hline NRZ-M & $=$ & Non-Return to Zero Mark \\
\hline ppm & $=$ & part per million \\
\hline RDD & $=$ & Return Data Delay \\
\hline $\mathrm{RF}$ & $=$ & Radio Frequency \\
\hline RPM & $=$ & Revolutions Per Minute \\
\hline RS & $=$ & Reed-Solomon \\
\hline $\mathrm{S} / \mathrm{C}$ & $=$ & Spacecraft \\
\hline SN & $=$ & Space Network \\
\hline SST & $=$ & Sub-Second Timer \\
\hline ST -5 & $=$ & Space Technology 5 \\
\hline$\mu s$ & $=$ & microsecond \\
\hline USNO & $=$ & US Naval Observatory \\
\hline UT & $=$ & Universal Time \\
\hline UTC & $=$ & Universal Time Coordinated \\
\hline UTCF & $=$ & Universal Time Correction Factor \\
\hline VEC & $=$ & Variable Emittance Controller \\
\hline
\end{tabular}




\section{1: BIOGRAPHIES}

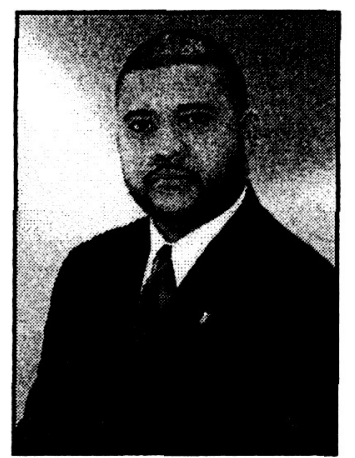

David Raphael is an electrical engineer at NASA/Goddard Space Flight Center's Flight Data Systems \& Radiation Effects Branch. He is currently the technical lead for the ST-5 C\&DH development. He began his career at NASA as an intern for two summers in the SICA (Summer Institute in Computer Applications) program in 1991 and 1992. From 1994-1996, he served as a technical lead at NASA's Wallops Flight Facility for all sounding rockets missions involving the MMP-900 encoder systems, for which he wrote a NASA Reference Publication. In 1996, he transferred to the Flight Development Group at Goddard where he worked on the Earth Orbiter -1 program, designing the processor board for the Wideband Advanced Recorder Processor (WARP) and supporting WARP development through board, box and spacecraft level I\&T and early orbit operations. He earned a Bachelor of Science in Electrical Engineering from Hofstra University in 1992, and a Master of Science in Electrical Engineering from the Johns Hopkins University in 1999.

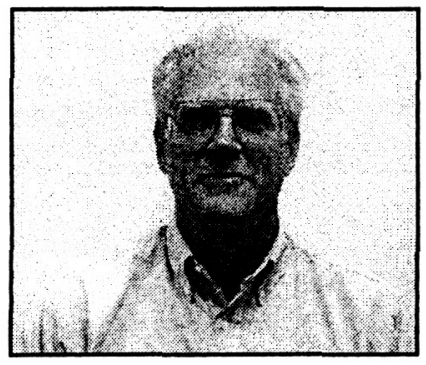

Victor Sank is a QSS contract engineer working at NASA/Goddard Space Flight Center's Communications Systems Branch. He is currently the lead designer for communications on the ST-5 satellites and for its transponder development. He began his career in medical physics imaging, having worked on X-ray computed tomography (CAT), positron emission tomography (PET) and magnetic resonance imaging (MRI). In 1985, when no new imaging technique was on the horizon, he made use of the computing and RF experience and turned to space communications and communications coding at the NASA/Goddard Space Flight Center. One of the first projects at the GSFC was the testing of the Comptom Gamma Ray Observatory (CGRO) spacecraft clock correlation system through the TDRSS. Documentation on the system was poor, requiring a fresh look that led to MOC software and the User Spacecraft Clock Correlation System Users Guide. He earned a Ph.D. in Physics from the Polytechnic University in 1971.

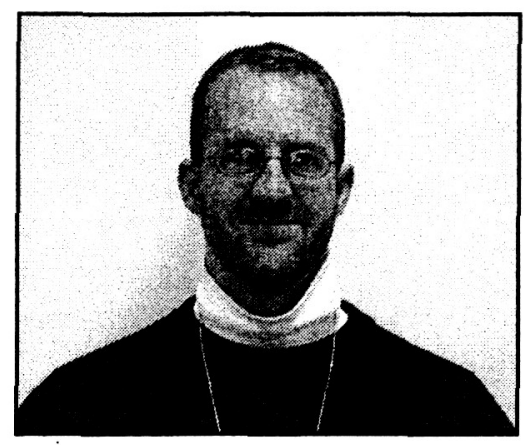

Philip Luers is the Associate Branch Head of the Flight Data Systems \& Radiation Effects Branch at NASA/Goddard Space Flight Center. Philip has been working the last 13 years developing electronics and data handling technologies and infusing them into flight missions. He has worked to infuse new technologies into flight missions including MAP, EO-1, TRMM, XTE, ACE, and SAMPEX. He participated in the timekeeping validation for MAP, EO-1, TRMM and XTE missions. He has technical oversight of the Branch's Flight Ethernet, Ultra Low Power, Spacewire, and Firewire developments. His past technology developments have included Essential Services Node, Mongoose V, Mongoose I, Rad-Hard FPGA, Fiber Optic Data Bus, Dual-rate 1773, and several others. He has served as Associate Branch Head for the last five years. He earned a Bachelor of Science in Electrical Engineering from the University of Maryland in 1988, and a Master of Science in Computer Science from the Johns Hopkins University in 1997.

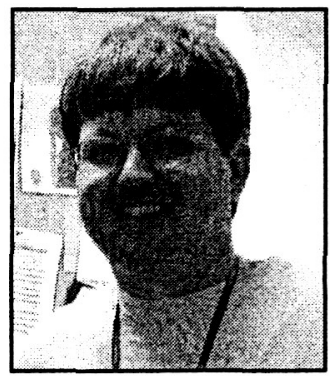

George Jackson is an electrical engineer at NASA/Goddard Space Flight Center's Flight Data Systems \& Radiation Effects Branch. He is currently the lead engineer for the ST-5 C\&DH development. He began his career at NASA's Wallops Flight Facility as a co-op student in 1991. From 1994 - 1995, he designed, built and tested aircraft data systems for airborne science projects at Wallops. In 1995, he transferred to the Radiation Effects and Analysis Group at Goddard where he conducted radiation testing on fiber optic and opto-electronics components and was lead engineer for a fiber optic experiment on the Naval Research Lab's Microelectronic and Photonic Test Bed. From 1997 2000, he worked on the Earth Orbiter -1 program, designing the science input board for the Wideband Advanced Recorder Processor (WARP) and supporting $W A R P$ development through board, box and spacecraft level I\&T and early orbit operations. He earned a Bachelor of Science degree in Physics from Salisbury State University in 1994 and a Master of Science degree in Electrical Engineering from George Washington University in 1999. 